\title{
Apoyo tecnológico para la fidelización y captación de nuevos clientes por medio de una aplicación móvil
}

\section{Technological Support for the loyalty and recruitment of new customers through a mobile implementation}

\section{Raúl Avellaneda}

Tecnólogo en Sistemas Fundación Universitaria Uninpahu, estudiante noveno semestre Ingeniería de Software Fundación Universitaria Uninpahu. Correo electrónico: javellanedaga@uninpahu.edu.co

Paulo André Martínez

Tecnólogo en Sistemas Fundación Universitaria Uninpahu, estudiante noveno semestre Ingeniería de Software Fundación Universitaria Uninpahu. Correo electrónico: pmartinezga@uninpahu.edu.co

\section{Sebastián Cabrera}

Tecnólogo en Sistemas Fundación Universitaria Uninpahu, estudiante noveno semestre Ingeniería de Software Fundación Universitaria Uninpahu. Correo electrónico: jcabrerasa01@uninpahu.edu.co

Carlos Gilberto Donoso Albarracín Magister en Docencia U. De La Salle, Docente asociado Fundación Universitaria Uninpahu. Correo electrónico: cdonosoal@uninpahu.edu.co

Información del artículo: recibido: agosto de 2016, aceptado: noviembre de 2016 https://doi.org/10.17081/invinno.5.1.2618 


\section{Resumen}

$\mathrm{L}$ a aparición de los teléfonos inteligentes ha revolucionado la vida. Los Palabras clave: teléfonos que antes solo se utilizaban para realizar llamadas y recibir mensajes, se han convertido en una herramienta de trabajo y de ocio indispensable. En este sentido se realizó una investigación cualitativa de Desarrollo, producto, teléfono, aplicación, cliente. tipo descriptivo, de la que se obtuvieron datos relevantes, evidenciando la oportunidad de desarrollar un producto a la medida de la compañía TU ESTILO RYE, para resolver la necesidad de incrementar la base de datos clientes. Para el equipo investigador, el teléfono inteligente se convirtió en la herramienta para fidelizar a los clientes con base en beneficios. La estrategia de la aplicación móvil se dirigió principalmente a apoyar la comunicación oportuna y directa con los clientes actuales, ampliando el control de acceso a una variedad mayor de instrumentos que les permitan realizar sus consultas de manera más real y oportuna. Una parte de las funcionalidades claves de la aplicación consiste en mostrar el saldo, el beneficio otorgado y la compra de artículos, sobre lo cual se precisa que una vez implementado el producto, se dará a conocer de forma personalizada a los clientes.

\section{Abstract}

A qualitative and descriptive research work was made here, of Keywords: which relevant data was obtained that evidenced the opportunity to develop a product fit to the size of the company TU ESTILO RYE, for solving the needs of increasing the database of its customers. development,product, phone,The appearance of smart phones, has changed drastically the way of life. In the past,phones were only used for making calls and receiving messages, today they have turned into a necessary working tool which is also used during your moments of relax and spare time. For the research team, the smart phone became a tool to keep customers by giving them benefits. The strategy of the mobile implementation has been mainly created to back up ready communications with current customers, widening access control to them with a greater variety of instruments that allow them to realize their queries in a more real and opportune way. As part of the key functions of this implementation is to disclose the balance, the benefits granted, and the purchase of goods. Once the product has been implemented, it will be communicated personally to each one of our customers. 


\section{INTRODUCCIÓN}

La aparición del sistema operativo Android supuso un antes y un después en lo que a la utilización de terminales móviles se refiere. No sería exagerado afirmar que, junto con su competidor más directo de Apple, Android ha revolucionado la forma de entender la telefonía móvil. Donde antes el usuario solo observaba un instrumento a través de llamadas telefónicas, ahora ese mismo usuario puede disponer de una herramienta potentísima que le permite mantener las mismas propiedades básicas de cualquier teléfono móvil, a la vez de disponer de todo un mundo de aplicaciones a su alcance de forma fácil y rápida.

Debido a la avanzada tecnología del mundo actual, se hace necesario una evolución constante, a fin de aprovechar las ventajas que está nos ofrece en cualquiera de los servicios donde se aplique. Pero poseer el manejo de la información en forma oportuna, rápida y sin límites, exige también el conocimiento general de la tecnología por parte de los clientes para su uso.

En efecto, tal y como se afirmó en el apartado precedente, una nueva forma de conexión con el mundo de las aplicaciones creadas para el sistema Android (no necesariamente en exclusiva), permite a los usuarios tener una presencia casi absoluta en multitud de servicios y productos, $y$, a las empresas que los producen, acceder a estos usuarios a través de una ventana asombrosamente rápida y más ventajosa de lo que se podrían haber imaginado antes.

Ahora bien, las aplicaciones se pueden clasificar por tema o utilidad. Esto le permite al usuario, en primer lugar, filtrar de antemano el servicio al que desea acceder, pudiendo orientarse más hacia el objetivo de su búsqueda: finanzas, juegos, herramien- tas para el propio terminal móvil, noticias, salud, meteorología, etc. De esta forma, se produce una primera aproximación del individuo con ese "mundo virtual" al que accede, y del que comienza a beneficiarse al ritmo que elija y con las preferencias que tenga [1].

En segundo lugar, es frecuente que la variedad de aplicaciones que se refieran a un mismo aspecto o tema sea elevada, hecho que puede resultar muy ventajoso para el usuario, pues, de esta forma, se le ofrece la posibilidad de elegir entre los diferentes criterios o preferencias que cada aplicación contiene, de manera que se le dé a cada aplicación el uso que se considere oportuno.

Por otra parte, con las aplicaciones móviles se puede operar desde un simple control de acceso hasta complejas combinaciones que pueden incluir la información personal, historial y cliente frecuente, incluyendo servicios financieros como saldos, abonos y beneficios.

El nivel de seguridad y la capacidad de almacenamiento y el procesamiento de información que se maneja en este sofisticado aplicativo facilita, además, la realización de procesos y permite administrar la información de mejor manera [2].

Por todo lo anterior, el objetivo de construir una aplicación móvil para dispositivos Android dirigido a los clientes de TU ESTILO RYE consiste en que la misma les permita conocer el saldo, beneficios y promociones de esta organización.

\section{METOdOLOGÍA}

Para cumplir con este propósito, se realizó un estudio acerca de la tecnología Android para el desarrollo de aplicaciones móviles y de la forma de cargar la aplicación móvil ya terminada con una estructura lógica para que facilite la navegación 
a los usuarios.

La metodología ingenieril utilizada para este proyecto fue la XP (Programación Extrema), y el objetivo concreto y principal consistió en desarrollar una aplicación móvil de calidad en tiempo y costo, así como la mejora continua del proceso para incrementar la eficacia y eficiencia de esta, y lograr así una alta productividad del equipo de desarrollo.

La metodología ágil para el desarrollo de software y para dispositivos móviles es conveniente para varios contextos, por ejemplo, la seguridad financiera, logística y aplicaciones de productos de simulación. Esta metodología ágil se basa en las prácticas de desarrollo de XP.

\section{Fases de la investigación}

Fase exploración

Esta fase se enfoca en planear y establecer el proyecto, es decir, en preparar y verificar todas las cuestiones relacionadas con el proyecto.

En concreto, se prueba la tecnología y se exploran las posibilidades de la arquitectura del sistema construyendo un prototipo. La fase de exploración toma poco tiempo en semanas dependiendo del tamaño.

Fase entrega

En esta fase el cliente establece la prioridad y los programadores realizan una estimación del esfuerzo. Se realizan acuerdos sobre el contenido de la primera entrega y se determina un cronograma en conjunto con el cliente.

\section{Fase iteración}

Incluye varias iteraciones sobre el sistema antes de entregarlo. El plan de entrega está compuesto por iteraciones de tres semanas. En la primera iteración se puede establecer una arquitectura del sistema para ser utilizada durante el proyecto.

\section{Fase producción}

Requiere pruebas adicionales y revisiones exhaustivas antes de que el sistema sea entregado al cliente. Al mismo tiempo, se deben tomar decisiones de inclusión de nuevas características a la versión actual durante la fase de mantenimiento.

\section{Fase mantenimiento}

Mientras la primera versión se encuentra en producción, el proyecto debe mantener el sistema en funcionamiento al mismo tiempo que se desarrolla nuevas iteraciones. La fase de mantenimiento puede requerir nuevo personal y cambios en su estructura.

\section{Fase muerte del proyecto}

Esto requiere que cumpla con lo requerido por el cliente como rendimiento $\mathrm{y}$ confiabilidad del sistema. Se genera la documentación final y no se realizan más cambios en la arquitectura [3].

\section{Descripción de la metodología seleccionada}

La Programación Extrema proporciona una serie de ventajas para la realización del proyecto, ya que ayuda al ahorro de tiempo y recurso.

Permite la entrega del producto usable con mayor rapidez, lo que permite la entrega de versiones continuas al usuario. Presenta un código sencillo y fácil de entender, que permite la reducción de errores en su 
desarrollo [4].

La respuesta oportuna ante cambios per-

Se decidió tomar como instrumentos mite la adaptación a nuevos requisitos del cliente.

Por estas y otras ventajas es la metodología que más se adecua a nuestro proyecto de desarrollo. de recolección de información la entrevista y la encuesta, que permitieron abordar de mucha mejor forma el estado actual del proceso desde el punto de vista de cada cliente.

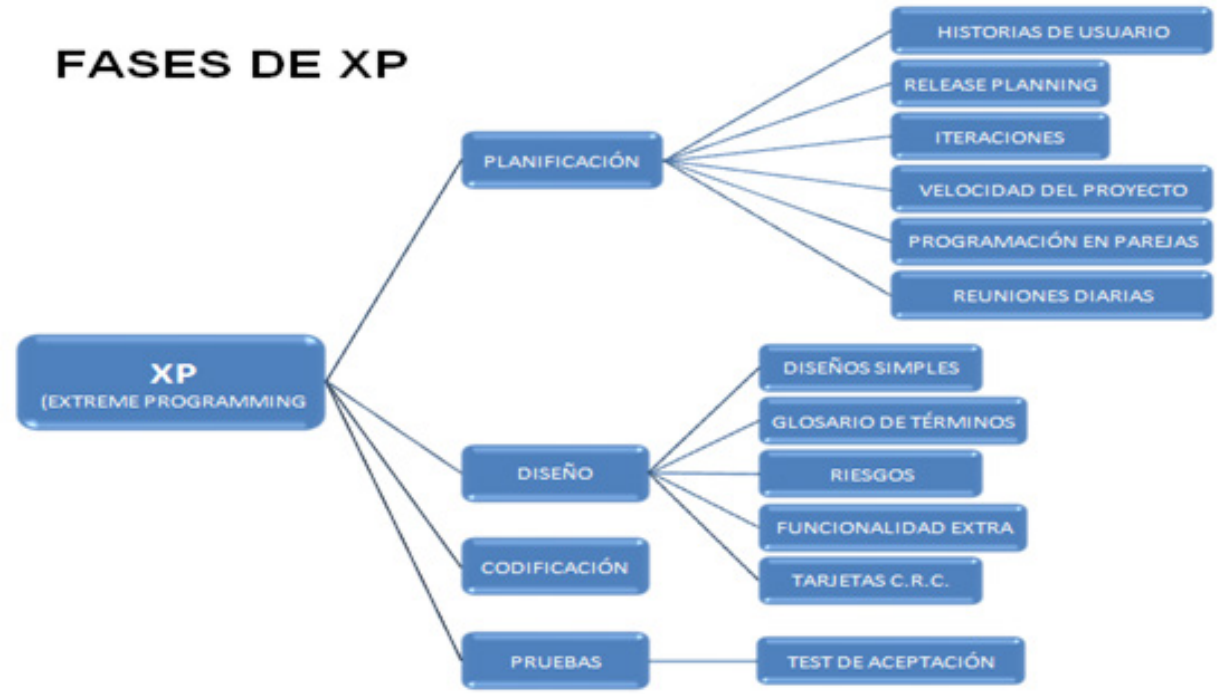

RESULTADOS

Después de aplicar los instrumentos de recolección de información, basados en la opinión de los encuestados acerca de la aceptación del móvil y sus beneficios se obtuvieron

Gráfica 1. Fases de un proyecto usando metodología XP

Fuente: [3].

los resultados, que se presentarán a continuación a partir de las preguntas y sus respectivas gráficas. Estas muestran el comportamiento de las respuestas dadas por los ciudadanos ya notificados.

Gráfica 2. Esquema de un proyecto usando metodología XP

Fuente: [3]. 


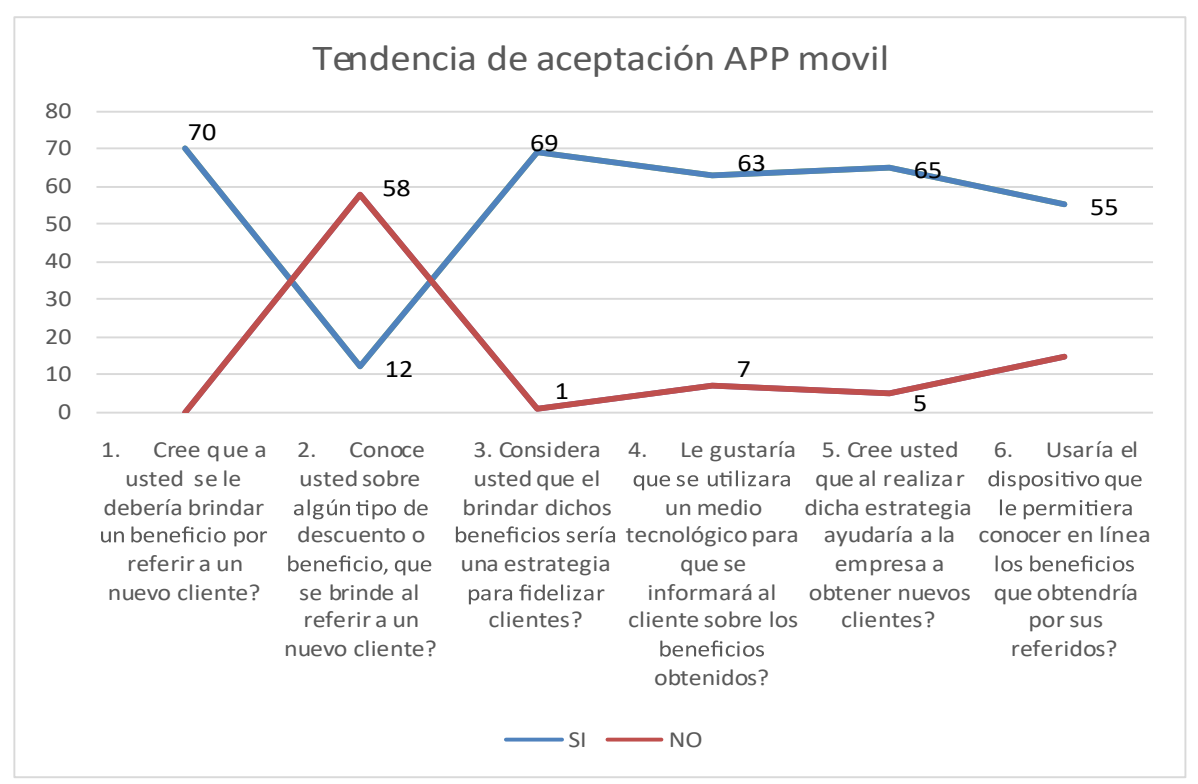

\section{Gráfica 3. Tendencias de aceptación aplicación móvil}

Fuente: Autores.

\section{Interpretación Gráfica 3}

Se tuvo la participación de 70 clientes activos, quienes contestaron una encuesta que consta de 5 preguntas de opción múltiple, con escala del 1 al 5, en la que 1 es "Excelente" y 5 es "Malo", así como con una pregunta abierta para sugerencias..

\section{Satisfaccion APP movil}

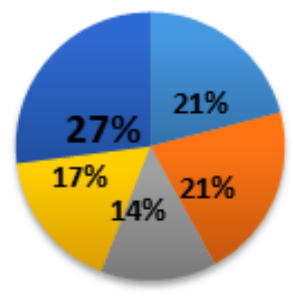

- Beneficios a futuras compras a Diseño

- Oportunidad de la infor mación = Veracidad del descuento

- Cobertura en la utilización

Gráfica 4. Nivel de satisfacción aplicación móvil Fuente Autores.

\section{Interpretación gráfica 4}

Actualmente, la tecnología es llamativa para el $100 \%$ de los clientes, lo que permite una gran aceptación de la propuesta de la aplicación móvil.

El $27 \%$ de los clientes prefiere el beneficio de cobertura en la utilización, y como segunda opción se ubica el diseño con un $21 \%$, así como los beneficios a futuras compras (también con un 21\%). Continúa en porcentaje de aprobación, la veracidad del descuento con $17 \%$. De todo esto se concluye que las personas aún no son conscientes de la importancia en la oportunidad de la información, la cual obtuvo el menor porcentaje $(14 \%)$, pero esto puede constituirse en una estrategia de mercado, ya que trabajando en forma dinámica en este punto se puede generar mayor atracción. 


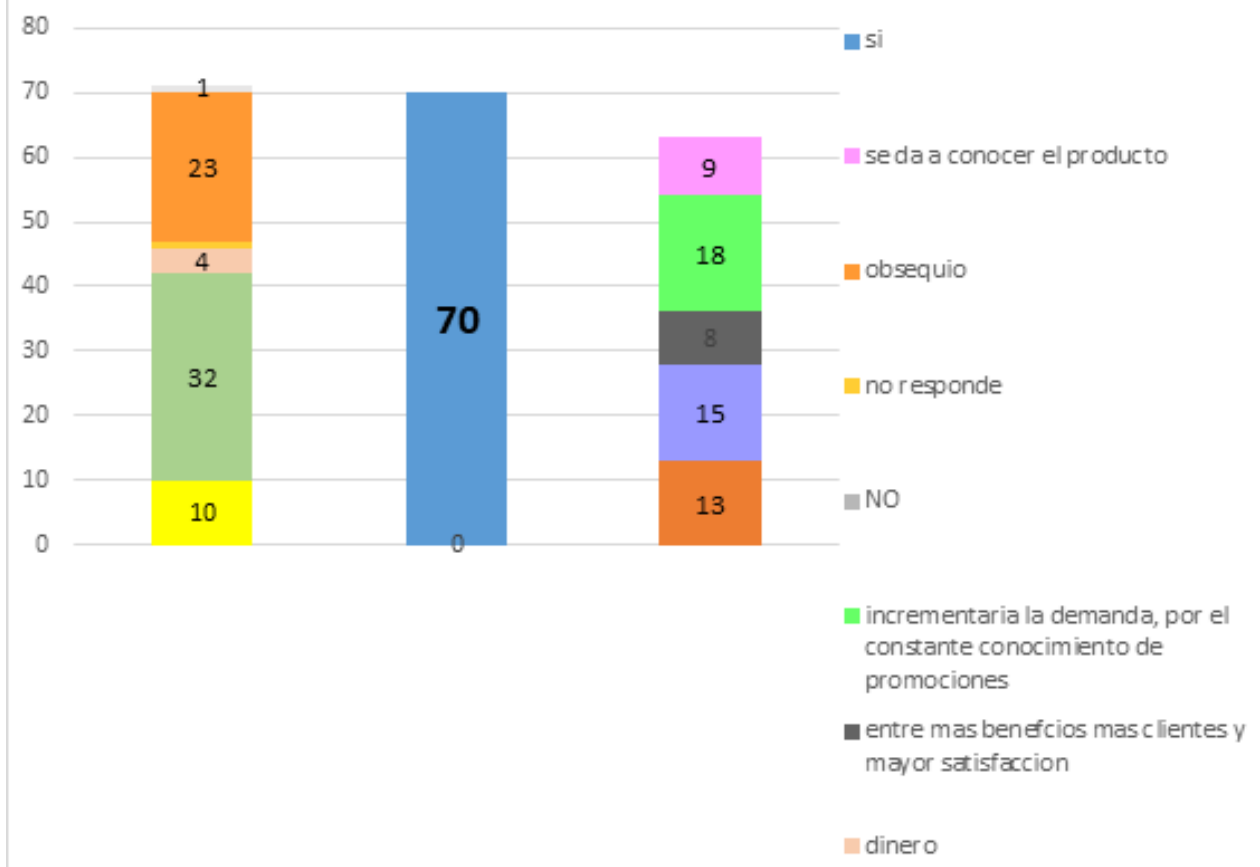

Gráfica 5. Efectividad de la información Fuente: Autores.

\section{Interpretación gráfica 5}

La tecnología es llamativa para el 100\% de los clientes, lo que permite una gran aceptación de la propuesta de la aplicación móvil. En concreto, atrae el recibir beneficios por referir a sus amigos, familiares, etc., y más cuando se trata de hacerlo por un medio que les pueda generar ganancias.

\section{DISCUSIÓN DE LOS RESULTADOS}

Dados los resultados anteriores, se puede afirmar que los dispositivos móviles cada día son más utilizados de manera distinta.

La aplicación móvil propuesta es innovadora, ya que por medio de esta se cumplirá el objetivo de incentivar a los clientes, brindando información de sus benefi- cios en tiempo real. Todos estuvieron de acuerdo en que, para que la información mejore, deben tener ayuda de una aplicación que les permita ingresar y mantener la información unificada, amén de que sea fácil de consultar.

Una de las principales razones por la que se desarrolló la aplicación en Android es, justamente, su alto nivel de utilización en plataformas celulares, pues, permite distribuir y modificar, ya que es un sistema completamente libre y a la vez el desarrollador puede decidir sobre su aplicación [5].

La aplicación móvil Android para la empresa TU ESTILO RYE se compone de varios módulos: Usuario, Producto, Solicitud de pedido, Estado de cartera consolidado, Estado de cartera detallado. A continuación se muestra el modelo Entidad Relación de la aplicación móvil, que estructura el almacenamiento de los datos propios del negocio: 


\section{DIAGRAMA ER APP TU ESTILO RYE}

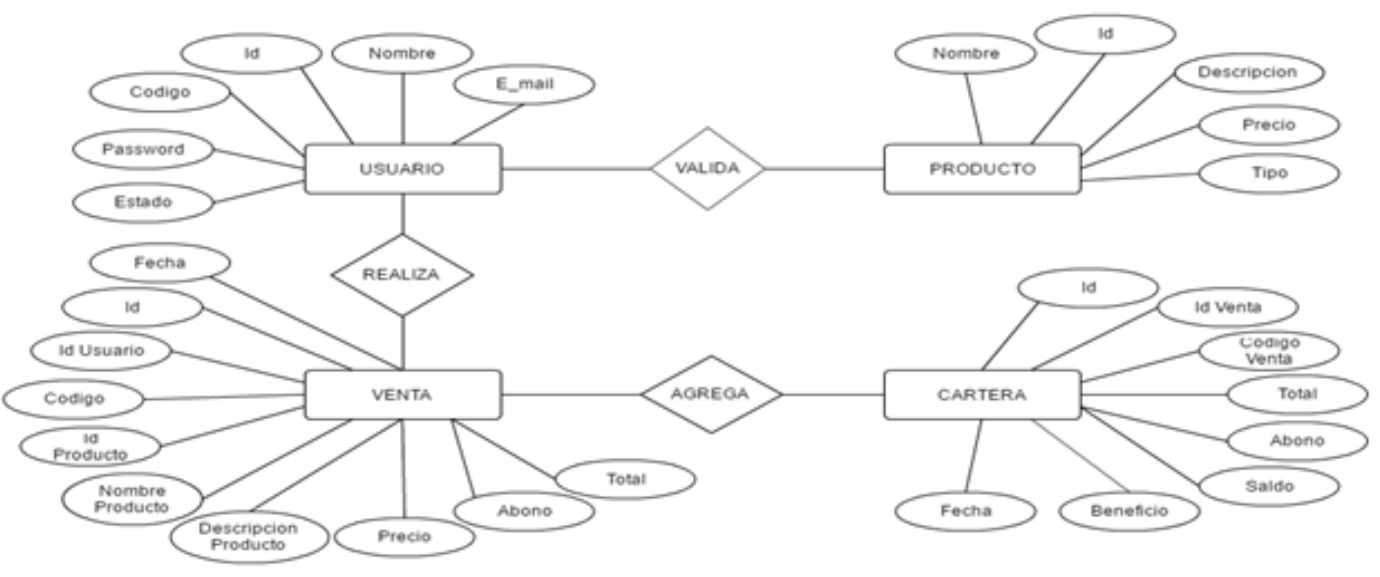

\section{Gráfica 6. Modelo Entidad Relación}

\section{Fuente: Autores.}

Arquitectura de la Aplicación

El proyecto se divide en dos partes. La primera es una aplicación para tu estilo RYE y para su funcionamiento se necesita de un dispositivo móvil Android, con la aplicación instalada y con acceso a Internet, por lo que se podrá consultar desde cualquier parte. Se halla conectada al servicio web, que a su vez se comunica con la base de datos de la empresa que almacena la información de pedidos.

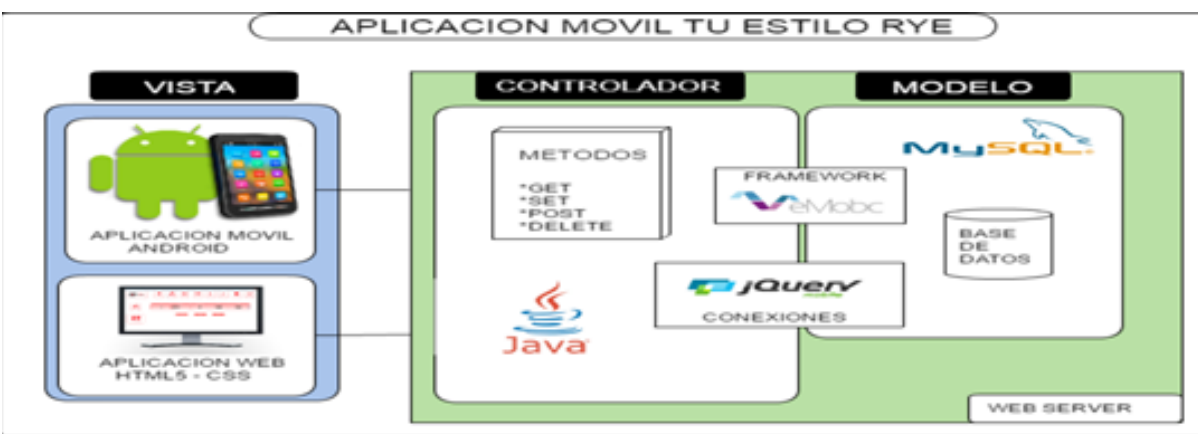

existente de la empresa, mediante peticiones $\mathrm{PHP}$, que interactúa con las peticiones del usuario HTLM5.

\section{Gráfica 7. Aplicación móvil Modelos Vista controlador}

Fuente: Autores.

La segunda parte del proyecto consta de un módulo web agregado a la intranet zar los servicios web para intercambiar datos en redes de ordenadores como Internet; en este caso, las organizaciones OASIS Y W3C son los responsables de la arquitectura y reglamentación de los servicios web 


\section{CONCLUSIONES}

El aplicativo elaborado fue satisfactorio para la empresa, ya que los requerimientos solicitados fueron diseñados de tal manera que se optimizaron los procesos de solicitud de pedidos, registro de los clientes e información sobre los estados de cartera. También se requirió el uso de una base de datos más segura en la que se realizaran las consultas de manera más rápida y por diferentes criterios, posibilitando la trazabilidad del estado de cartera sin pérdida de información.

Con este producto de desarrollo se genera así una oportunidad de negocio que propicia la expansión de la base de clientes y que es replicable a empresas pequeñas y medianas con intereses similares.

La exploración de las nuevas tecnologías, como la presente, hará posible que se consoliden cada vez más diversas estrategias de fidelización de clientes, con valores agregados que masifiquen su uso en beneficio de los mismos clientes y de los pequeños empresarios.

Finalmente, es importante manifestar que los escenarios de crecimiento de las pequeñas y medianas empresas se están consolidando a medida que los avances tecnológicos se hacen visibles en acciones concretas y eficientes, generando oportunidades para satisfacción a los clientes, y conllevando a una expansión de mercado basada en una mejor experiencia de compra.

\section{REFERENCIAS}

[1]. Movil.Blogspot. (AES)FederalInformation. ProcessingStandardsPublication. 2015.

[2]. Processing Standards Publication. Data Encryption Standard. 1999.

[3]. E. Jeffries Ronald. What is Extre- me Programming? [Que es Programación Exterma?]. [en línea].1999-2013.Disponible en internet < http://xprogramming.com/ what-is-extreme-programming/>

[4]. S. Hashimi, S. Komatineni. "Pro Android, Apress". 2009

[5]. S. Hashimi, S. Komatineni, D. MacLean. "Pro Android 2, Apress". 2010.

Bibliografìa de consulta

F. Ableson, R. Sen, C. King. "Android in Action, Manning Publications". 2011.

S. CardGroup. Smart Card Tutorial, 1992.

Z. Craig. Manual de Referencia.

R. Duque Hoyos. "Disciplinariedad, interdisciplinariedad, y transdisciplinariedad - Vínculos y límites. (I. D. Carmona Aranzazu, Ed.)". Escritos, vol. 14 n$^{\circ} .32$, pp. 94-125. 2006.

P. B. Blanc, Le PôleSécurité. En Le PôleSécurité (pág. 27). Publication des principauxdocuments de l'année., 2000.

D. Groth, \& T. Skandier. Guia del estudio de redes (4a edición ed.). Sybex Inc. 2005.

S. Haykin, Introduction to Signals and Systems (Wiley, Ed.) histinf.blogs. (11 de 04 de 2011). historia-de-las-bases-de-datos. J. M. Rodriguez. Métodos de investigación cualitativa. 2011.

S. Taylor. Introducción a los métodos cualitativos de investigación la búsqueda de significados. Buenos Aires: Paidós. 1998. 


\section{Este artículo se cita:}

R. Avellaneda, S. Cabrera, P. Martínez, y C. Donoso , "Apoyo tecnológico para la fidelización y captación de nuevos clientes por medio de una aplicación móvil", Revista Investigación e Innovación en Ingenierias, vol. 5, n'. 1, pp. 92-101, 2017. 